\title{
GENERALIZED TIME-BANDWIDTH PRODUCT OPTIMAL SHORT-TIME FOURIER TRANSFORMATION
}

\author{
Lütfiye Durak and Orhan Arıkan \\ Department of Electrical Engineering, Bilkent University, Ankara, 06533 Turkey \\ Phone: 90-312-2664307, Fax: 90-312-2664192 \\ E-mail: lutfiye@ee.bilkent.edu.tr; oarikan@ee.bilkent.edu.tr
}

\begin{abstract}
By extending the time-bandwidth product concept to fractional Fourier domains, a generalized time-bandwidth product (GTBP) is introduced. The GTBP provides a rotation independent measure for the support of the signals in time-frequency domain. A close form expression for the adaptive kernel of STFT that provides the minimum increase on the GTBP of a signal is derived. Also, a linear canonical decomposition of the obtained GTBP optimal STFT is presented to identify its relation to the rotationally invariant STFT analysis.
\end{abstract}

\section{INTRODUCTION}

The choice of the STFT kernel determines the time-frequency (TF) signal localization properties of the distribution. Among the Hermite-Gaussian function family that satisfies the T-F domain rotation property, the gaussian function is the most commonly used kemel function. This is mainly because of the fact that the gaussian kernel has the minimum time-bandwidth product (TBP). However, STFT with the gaussian kernel still suffers from the problem of limited resolution. To overcome the inherent trade-off between the time and the frequency localization of the STFT, several alternatives have been investigated in the literature $[1,2]$. Although these investigations provide significant improvements in the T-F localization of signal components, in the presence of chirp-like signals, they still provide descriptions whose localization properties depend on the orientation of the signal components in the T-F domain.

In this paper, we introduce a generalization of the TBP, GTBP, to obtain a measure of compactness in the T-F plane that does not change even if the signal is fractionally Fourier transformed, or equivalently, the signal is rotated in the T-F plane. Then, we obtain the optimal STFT kernel which provides the most compact representation considering the GTBP of a signal component. The proposed T-F analysis is shown to be equivalent to an ordinary STFT analysis conducted in a scaled fractional Fourier transform domain. The obtained GTBP optimal STFT representation yields optimally compact T-F supports for chirp-like signals on the STFT plane. To provide further insight, a linear canonical decomposition of the obtained GTBP optimal STFT analysis is presented. The canonical decomposition reveals that GTBP optimal STFT is equivalent to cascade connections of signal-independent T-F domain rotation and scaling operations and signal-independent STFT analysis with the zeroth-order Hermite-Gaussian kemel.

\section{TIME-FREQUENCY LOCALIZATION BY STFT}

An important criterion for the success of T-F representations is how well it preserves the T-F domain support of signals. Among the commonly used T-F representations, WD is the best in this respect. However, the cross-terms of the WD clutters the obtained T$F$ representation. Therefore, in a way it disturbs the actual support of the signal in the T-F domain. The STFT family provides crossterm free T-F representations. So, support preservation criteria is applicable to measure the success of the alternative STFT representations. In this section we investigate the effect of the STFT kemel on the obtained T-F support of the signal components. This investigation will require generalized definition of the TBP. Furthermore, it will lead to signal adaptive STFT's.

The T-F domain support of a signal $x(t)$ is commonly measured by its time width, $T_{x}$, and its frequency domain bandwidth, $B_{x}$. Therefore the TBP, which is defined as $T_{x} \cdot B_{x}$, has been commonly used as a measure for the T-F domain support of the signal. The well known uncertainty principle dictates that $1 / 4 \pi$ is a lower bound on the TBP of a signal and only the gaussian signal has a TBP equal to this lower bound [3]. If the TBP is chosen as the measure of support, a well defined optimization problem can be cast for the optimal STFT kernel as presented in Appendix A.

Although the TBP of a signal is commonly used, it is not a satisfactory measure for the T-F support of signals. This is illustrated in Fig. 1 -(a), where support of a chirp signal and its bounding rectangle with sides time and frequency widths and area equal to TBP is shown. For a windowed chirp signal $x(t)=$ $e^{j \pi\left(\alpha t^{2}+2 \beta t\right)} e^{-\pi \gamma t^{2}}$, the TBP of the signal $x(t)$ equals to:

$$
\operatorname{TBP}\{x(t)\}=\frac{1}{4 \pi}\left[1+\frac{\alpha^{2}}{\gamma^{2}}\right]^{1 / 2} .
$$

Even though the T-F domain rotation operation that is implemented by fractional Fourier transformation (FrFT) just rotates the support of $x(t)$, the TBP shown in Fig. 2 changes significantly. The minimum TBP is reached when the signal support is oriented along the time or frequency axes. As seen from this example, TBP is always an upper bound to the support of the signal in the T-F domain. To obtain a tighter measure, here we propose a generalization of TBP which is defined as:

$$
\operatorname{GTBP}\{x(t)\}=\min _{0 \leq a<4} \operatorname{TBP}\left\{x_{a}(t)\right\}
$$

where $x_{a}$ is the $a^{t h}$ order FrFT of $x(t)$ given by [3]:

$$
x_{a}(t) \equiv\left\{\mathcal{F}^{a} x\right\}(t)=\int B_{a}\left(t, t^{\prime}\right) x\left(t^{\prime}\right) d t^{\prime}, 0<|a|<2
$$




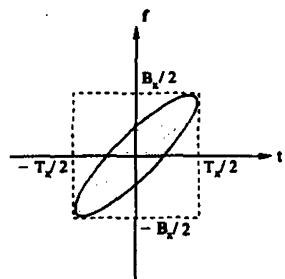

(a)

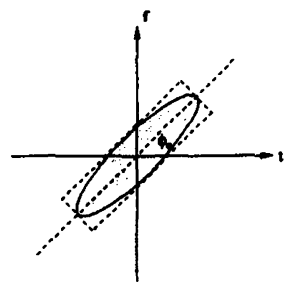

(b)
Fig. 1. TBP which is the area of the dashed rectangle shown in (a) is not a tight measure for the T-F support of signals. The GTBP is the area of the tightest bounding rectangle to the support of the signal as illustrated in (b).

where $a \in \Re$ and $B_{a}\left(t, t^{\prime}\right)$ is:

$B_{a}\left(t, t^{\prime}\right)=\frac{e^{-j\left(\frac{\pi}{4} \operatorname{sgn}(\sin \phi)+\frac{\phi}{2}\right)}}{|\sin \phi|^{1 / 2}} e^{j \pi\left(t^{2} \cot \phi-2 t t^{\prime} \csc \phi+t^{\prime 2} \cot \phi\right)}$

The generalized definition satisfies the fundamental rotation invariance property, so that the GTBP of a signal $x(t)$ and its $a_{0}^{t h}$ order FrFT, $x_{a_{0}}(t)$ is the same for any $a_{0}$. This can be proved as follows. Since the FrFT is order additive, the GTBP of $x_{a_{0}}(t)$ is:

$$
\operatorname{GTBP}\left\{x_{a_{0}}(t)\right\}=\min _{0 \leq a<4} \operatorname{TBP}\left\{x_{a_{0}+a}(t)\right\} .
$$

Since the fractional Fourier transformation is periodic with a period of 4, we obtain:

$$
\operatorname{GTBP}\left\{x_{a_{0}}(t)\right\}=\min _{0 \leq a<4} \operatorname{TBP}\left\{x_{\left(\left(a_{0}+a\right)\right)_{4}}(t)\right\}
$$

where $((\cdot))_{4}$ denotes the modulo by 4 operation. Now by changing the variable of minimization to $a^{\prime}=\left(\left(a_{0}+a\right)\right)_{4}$, we get:

$$
\operatorname{GTBP}\left\{x_{a_{0}}(t)\right\}=\min _{0 \leq a^{\prime}<4} \operatorname{TBP}\left\{x_{a^{\prime}}(t)\right\}
$$

which is equal to the GTBP $\{x(t)\}$ given in (2). Thus, for any $a_{0}$ :

$$
\operatorname{GTBP}\left\{x_{a_{0}}(t)\right\}=\operatorname{GTBP}\{x(t)\} \text {. }
$$

As illustrated in Fig. 1-(b), the GTBP provides the tightest bounding rectangle to the support of the signal in the T-F domain. As it is investigated for the TBP in Appendix A, it is important to obtain the optimal STFT kernel considering the GTBP of a signal. Actually, this investigation can be conducted as follows. As shown in Fig. 1-(b), for a signal $x(t)$ whose bounding rectangle is oriented at an angle $\phi_{0}$, which is not equal to 0 or $\pi / 2$, the fractionally Fourier transformed signal $x_{a 0}(t)$ where $a_{0}=\frac{2}{\pi} \phi_{0}$ has its bounding rectangle oriented along the time axis. The transformed signal $x_{a_{0}}(t)$ has its TBP and GTBP equal to each other. Therefore for $x_{a_{0}}(t)$ the optimal STFT window is the gaussian window given in (18) with $T_{x_{a_{0}}}$ and $B_{x_{a_{0}}}$ as the corresponding time and bandwidth of the transformed signal. The desired T-F representation of $x(t)$ can be obtained as the counter-clockwise rotation of the optimal STFT for $x_{a_{0}}(t)$ by an angle of $\phi_{0}$. Since the STFT of $x_{a_{0}}(t)$ is:

$$
S_{x_{a_{0}}}(t, f)=\int x_{a_{0}}\left(t^{\prime}\right) h^{*}\left(t^{\prime}-t\right) e^{-\jmath 2 \pi f t^{\prime}} d t^{\prime},
$$

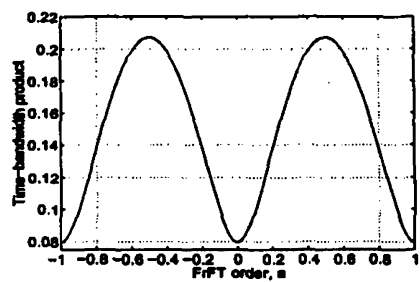

Fig. 2. The rotation operation does not change the area of the support of a chirp signal $x(t)=e^{j \pi\left(5 t^{2}+2 \beta t\right)} e^{-\pi t^{2}}$, however the TBP changes. The dependence of the TBP on the FrFT order $a$ is shown for $x(t)$.

by using (3) and the property $B_{a}\left(t^{\prime}, \tau\right)=B_{-a}\left(t^{\prime}, \tau\right)$ we obtain:

$S_{x_{a_{0}}}(t, f)=\int\left[\int h\left(t^{\prime}-t\right) e^{j 2 \pi f t^{\prime}} B_{-a_{0}}\left(t^{\prime}, \tau\right) d t^{\prime}\right]^{*} x(\tau) d \tau$ (10) with optimal gaussian kernel $h(t)=e^{-\pi \gamma t^{2}}$ where $\gamma=\frac{B_{a_{a_{0}}}}{T_{\boldsymbol{s}_{0}}}$. Then the desired representation of $x(t)$ is equal to $R_{\phi_{0}}\left\{S_{x_{a_{0}}}(t, f)\right\}$ and can be written as:

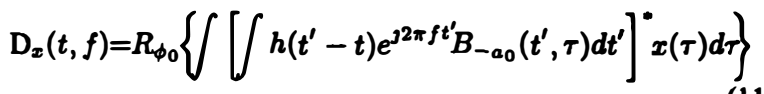

In (11), the expression in the brackets can be recognized as the $-a_{0}^{t h}$-order FrFT of $h\left(t^{\prime}-t\right) e^{j 2 \pi f t^{\prime}}$ which is simply the time and frequency shifted form of the kernel $h(t)$. Using the time and frequency shift properties of the FrFT, $D_{x}(t, f)$ is obtained as:

$$
\mathrm{D}_{x}(t, f)=e^{-\jmath \pi \psi} \int x(\tau) g_{G T B P}^{*}(\tau-t) e^{-\jmath 2 \pi f \tau} d \tau
$$

where $\psi=\left(t^{2}-f^{2}\right) \sin \phi_{0} \cos \phi_{0}+2 t f \sin ^{2} \phi_{0}$, and the optimal kernel is:

$$
g_{G T B P}(\tau)=K e^{-J \pi \tau^{2} \frac{\cot \phi_{0}\left(\gamma^{2}-1\right)}{\gamma^{2}+\cot ^{2} \phi_{0}}} e^{-\pi \tau^{2} \frac{\gamma \operatorname{coc}^{2} \phi_{0}}{\gamma^{2}+\cot ^{2} \phi_{0}}}
$$

where $K=\sqrt{\frac{1+y \cot \phi_{0}}{\gamma+j \cot \phi_{0}}}$. Since the phase $\psi$ can be ignored in $D_{x}(t, f)$, it is easy to see that the desired representation in (12) has the form of STFT with kemel $g_{G T B P}(\tau)$. This explicit form of the GTBP optimal STFT distribution provides significant computational saving in practice. Once the fractional order $a_{0}$, and the corresponding time-width $T_{x_{a_{0}}}$ and the bandwidth $B_{x_{a_{0}}}$ of the signal in this fractional domain is determined, the computational complexity of (12) is the same as the computational complexity of the ordinary STFT.

In Fig. 3, T-F domain localization by TBP optimal STFT and GTBP optimal STFT of a chirp signal, $x(t)=e^{\pi t^{2}-\pi t^{2} / 9}$ is compared. T-F support of the GTBP optimal STFT illustrated in Fig. 3-(b) is significantly better localized when compared to the the TBP optimal STFT illustrated in Fig. 3-(a). To illustrate the effect of noise, a synthetic chirp signal embedded in $-5 \mathrm{~dB}$ noise is analyzed by both TBP and GTBP optimal STFT as shown in Fig. 4. A significant improvement for the T-F localization of chirplike components is observed when compared to the ordinary STFT with similar computational complexity. In the next section, we will provide a canonical decomposition of GTBP optimal STFT such that the rotation invariance is satisfied in a certain scaled fractional Fourier domain. 


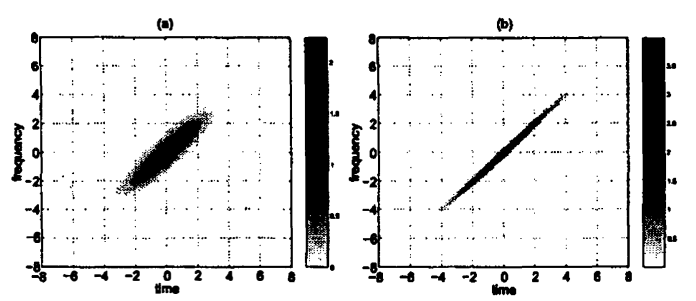

Fig. 3. The GTBP optimal STFT illustrated in (b) has a significantly improved T-F support than the TBP optimal STFT illustrated in (a).

\section{GTBP OPTIMAL STFT AND ROTATION PROPERTY}

Although (12) provides an efficient implementation method for the GTBP optimal STFT analysis, we would like to introduce a multistage implementation of it as shown in Fig. 5-(b). This decomposition is a linear canonical representation of the GTBP optimal STFT analysis with a sequence of operations explained and illustrated in Fig. 6. First, the $a_{0}^{t h}$ order FrFT of the input signal is computed using (3). As shown in Fig. 6-(d), the T-F support of the transformed signal (shown in Fig. 6-(c)) is oriented along the time axis. Then the transformed signal is scaled so that the TF support will fit into a circular support. The scaling parameter $M$ is chosen as $\sqrt{B_{x_{0}} / T_{x_{0}}}$ [4]. As shown in Fig. 6-(f), the T-F support of the scaled signal (shown in Fig. 6-(e)) fits into a circular region. Once the support of the signal becomes circular, the GTBP optimal STFT and the TBP optimal STFT becomes identical. Hence, as it can be shown easily by using (18), the kernel of the GTBP optimal STFT is the zeroth-order Hermite-Gaussian function $h_{0}(t)=e^{-\pi t^{2}}$. Such an STFT operation satisfies the following rotation property:

$$
\left|S_{x_{a_{0}}}(t, f)\right|=R_{-\phi_{0}}\left\{\left|S_{x}(t, f)\right|\right\}, \phi_{0}=a_{0} \pi / 2
$$

where $R$ denotes for the counter-clockwise rotation operator. This can be shown as follows. If $h_{0}(t)$ is used as the kernel function of the $S_{x_{a_{0}}}(t, f)$ in (10), by employing the time and frequency shift properties of the FrFT [3], we obtain:

$$
\begin{aligned}
S_{x_{a_{0}}}(t, f) & =e^{\jmath \psi} \int x(\tau) h_{0}^{*}\left(\tau-t \cos \phi_{0}+f \sin \phi_{0}\right) \\
& \times e^{j 2 \pi \tau\left(t \sin \phi_{0}+f \cos \phi_{0}\right)} d \tau
\end{aligned}
$$

where $\psi=\left(t^{2}-f^{2}\right) \sin \phi_{0} \cos \phi_{0}+2 t f \sin ^{2} \phi_{0}$. The magnitude of $S_{x_{a_{0}}}(t, f)$ in (15) is simply equal to the magnitude of $R_{-\phi_{0}}\left\{S_{x}(t, f)\right\}$ satisfying the rotation property stated in (14).

The obtained STFT representation with the zeroth-order Hermite-Gaussian kernel should be operated in two successive stages to provide the final answer. First, a T-F domain scaling should be performed so that,

$$
D_{s}(t, f)=D(t / M, M f)
$$

where $D(t, f)$ is the input and $D_{s}(t, f)$ is the output of the T-F scaling operation. The effect of this scaling operation is shown in Fig. 6-(f) and (g). Then, the final GTBP optimal STFT distribution is obtained by the rotation operation, $R_{\phi}\left\{D_{s}(t, f)\right\}$ and its effect is shown in Fig. 6-(h) which yields a high-resolution T-F description corresponding to the original signal.
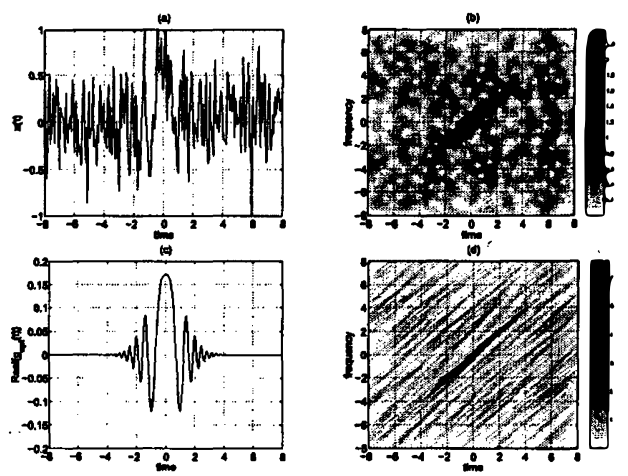

Fig. 4. The noisy chirp signal shown in (a) is analyzed by both the TBP optimal STFT illustrated in (b) and the GTBP optimal STFT illustrated in (d) with the kernel function shown in (c).

The main reason behind the introduction of this canonical decomposition is that the rotation invariant STFT with the zerothorder Hermite-Gaussian kernel is explicitly shown to be part of every GTBP optimal STFT analysis. Therefore, for any arbitrary mono-component signal, there exists a "natural domain" where the rotation independent STFT analysis with zeroth-order HermiteGaussian kernel provides the GTBP optimal STFT representation. The signals are transformed to their "natural domains" by the first two operations of the canonical decomposition. We believe this concept of "natural domain" is theoretically significant and will provide further insight to the research on T-F signal analysis.

\section{CONCLUSIONS AND FUTURE WORK}

In this paper, we introduced the GTBP which provides a rotation invariant measure for the T-F support signals. Then, the GTBP optimal STFT analysis is proposed for mono-component signals. Along with the proposed efficient implementation of the GTBP optimal STFT, a theoretically insightful canonical decomposition of it is presented. This way, the GTBP optimal STFT analysis is related to the rotationally invariant STFT analysis with the zeroth order Hermite-Gaussian kernel. Also, this decomposition provided a "natural domain" concept for mono-component signals.

The proposed GTBP optimal STFT requires determination of parameters related to the T-F support of mono-component signals which must be adaptively chosen in practical applications [5]. Also, the GTBP optimal STFT representation of multi-component signals needs the determination of the required set of parameters for each signal component. Efficient ways of estimating the required support parameters require further research.

\section{APPENDIX A}

In this appendix, TBP optimal STFT analysis is presented. As shown in Fig. 7, for an STFT kernel $g(t)$, the T-F domain support of the representation for $x(t)$ can be zoned into a rectangular region of respective T-F dimensions of $\left(T_{x}+T_{g}\right)$ and $\left(B_{x}+B_{g}\right)$. To choose the optimal window that adapts to the analyzed signal $x(t)$, the following optimization criterion can be used:

$$
\min _{T_{g}, B_{g} ; T_{g} \cdot B_{g} \geq \frac{1}{4 \pi}}\left(T_{x}+T_{g}\right) \cdot\left(B_{x}+B_{g}\right)
$$




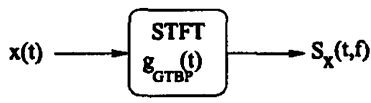

(a)

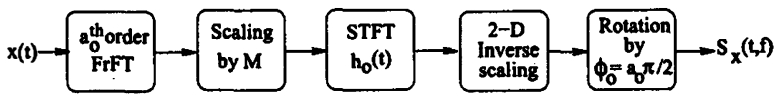

(b)

Fig. 5. Block diagram of GTBP optimal STFT is shown in (a), and the linear canonical decomposition of it is shown in (b). The first two operational blocks in (b) transforms the mono-component signal $x(t)$ to its "natural domain" where the rotation invariant STFT with kernel $h_{0}(t)$ provides the GTBP optimal STFT distribution.

It can be easily seen that the optimal solution $g(t)$ must satisfy the uncertainty principle with equality, therefore, it must be a gaussian kernel. Hence, the optimization problem can be solved in this set by just obtaining the time-width of the gaussian by solving the following problem:

$$
\min _{T_{g}}\left(T_{x}+T_{g}\right) \cdot\left(B_{x}+\frac{1}{4 \pi} \frac{1}{T_{g}}\right)
$$

With a little effort the optimal time-width of $g(t)$ is obtained as $T_{g}=\sqrt{\frac{T_{x}}{4 \pi B_{x}}}$ and the corresponding optimal gaussian window is:

$$
g_{T B P}(t)=e^{-\pi t^{2} B_{x} / T_{x}} .
$$

For $x(t)$ itself a gaussian signal with $T_{x} \cdot B_{x}=1 / 4 \pi$, this optimal time-support for the gaussian kernel function reduces to the commonly used rule-of-thumb of choosing the $T_{g}=T_{x}$. Otherwise, the optimal time support $T_{g}$ is always shorter than the time support of the analyzed signal $T_{x}$.

\section{REFERENCES}

[1] E. J. Rothwell, K. M. Chen, and D. P. Nyquist, “An adaptivewindow-width short-time Fourier transform for visualization of radar target substructure resonances," IEEE Trans. Antennas and Propagation, vol. 46, no. 9, pp. 1393-1395, Sept. 1998.

[2] G. Jones and B. Boashash, "Generalized instantaneous parameters and window matching in the time-frequency plane," IEEE Trans. Signal Process., vol. 45, no. 5, pp. 1264-1275, May 1997.

[3] H. M. Ozaktas, Z. Zalevsky, and M. A. Kutay, The Fractional Fourier Transform with Applications in Optics and Signal Processing, John Wiley \& Sons, 2000.

[4] H. M. Ozaktas, O. Arikan, M. A. Kutay, and G. Bozdagi, "Digital computation of the fractional Fourier transform," IEEE Trans. Signal Process., vol. 44, no. 9, pp. 2141-2150, Sept. 1996.

[5] A. K. Özdemir, L. Durak, and O. Ankan, "High resolution time-frequency analysis by fractional domain warping," Proc. IEEE Int. Conf. Acoust. Speech Signal Process., May 2001.
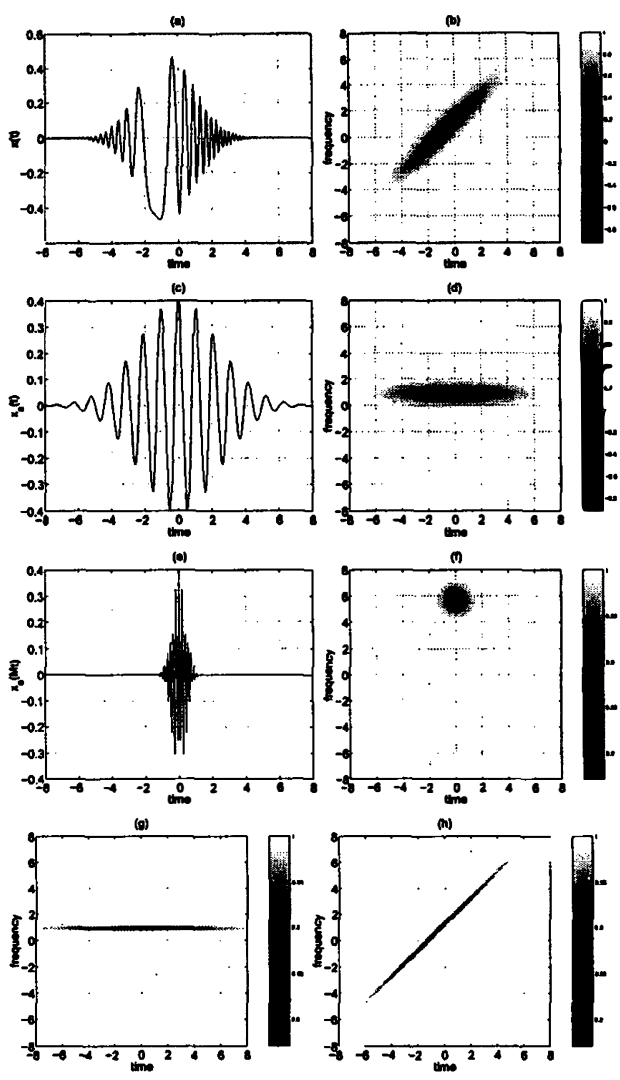

Fig. 6. For a chirp-like signal shown in (a), the FrFT is computed so that the chirp is converted to a sinusoidal as in (c). The corresponding STFT's are shown in (b) and (d), respectively. Through appropriate scaling $x_{a}(t)$ is converted to a zeroth-order HermiteGaussian enveloped sinusoidal as illustrated in (e) and its STFT is computed with the gaussian window as shown in (f). This is followed by 2-D scaling which inverts the scaling on the signal as shown in (g), and finally the distribution is rotated back to its original orientation removing the FrFT effect as illustrated in (h).

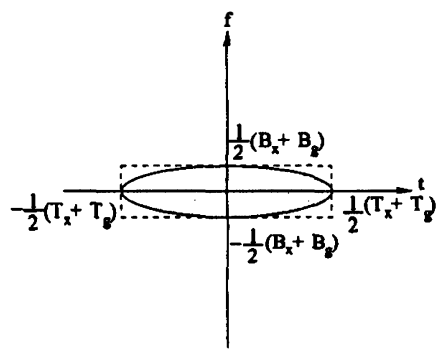

Fig. 7. The T-F domain support of the STFT representation for $x(t)$ with kernel $g(t)$ can be zoned into a rectangular region of respective T-F dimensions of $\left(T_{x}+T_{g}\right)$ and $\left(B_{x}+B_{g}\right)$. 